\title{
Engartner, Tim, Reinhold Hedtke, und Bettina Zurstrassen (2021): Sozialwissenschaftliche Bildung. Politik - Wirtschaft - Gesellschaft
}

\author{
Paderborn: Verlag Ferdinand Schöningh. 278 Seiten. 25,00 €
}

\section{Marc Partetzke}

Angenommen: 31. März 2021 / Online publiziert: 21. April 2021

(C) Der/die Autor(en) 2021

Mit ihrem Lehrbuch „Sozialwissenschaftliche Bildung“ bedienen Tim Engartner, Professor für Didaktik der Sozialwissenschaften an der Goethe-Universität Frankfurt, Reinhold Hedtke, Seniorprofessor am Fachbereich Gesellschaftswissenschaften derselben Universität, und Bettina Zurstrassen, Professorin für Didaktik der Sozialwissenschaften an der Universität Bielefeld, zweifelsohne ein Desideratum. So ist eine „Einführung“ in die sozialwissenschaftliche Bildung, die als ein „Studienund Arbeitsbuch" angelegt ist, aus Sicht der anvisierten Zielgruppe - „Studierende, Lehrende sowie Referendarinnen und Referendare von Unterrichtsfächern wie ,Politik und Wirtschaft', ,Sozialwissenschaften', ,Arbeitslehre', ,Wirtschaft-ArbeitTechnik', ,Verbraucherbildung ' sowie ,Gemeinschaftskunde“ und ,Sozialkunde' der allgemein- und berufsbildenden Schulen, aber auch [...] Lehrende in außerschulischen (politischen) Bildungseinrichtungen“ (S. 17) - längst überfällig gewesen. In insgesamt fünf Kapiteln liefern die Autor*innen nicht nur ein fachdidaktisches Propädeutikum, sondern auch zahlreiche „Unterrichtsmaterialien, Quellentexte und Anregungen zur Unterrichtsgestaltung“ (S. 17). Beantworten wollen sie ferner die Fragen:

- „was sozialwissenschaftliche Bildung kennzeichnet,

- welche Charakteristika die Sozialwissenschaften ausmachen,

- welchen erheblichen Mehrwert die interdisziplinäre Auseinandersetzung mit sozialwissenschaftlichen Wissensbeständen und Werkzeugen eröffnet und

- welche Möglichkeiten sich bieten, sozialwissenschaftliche Bildung zu organisieren“" (S. 12).

M. Partetzke ( $\triangle)$

Stiftung Universität Hildesheim, Hildesheim, Deutschland

E-Mail: partetzke@uni-hildesheim.de 
Außerdem soll ihr Buch ,zur Auseinandersetzung und zum Nachdenken über sozialwissenschaftliche Bildung animieren“ und „Reflexionsprozesse über das eigene Professionsverständnis anregen" (S. 17). So werden im ersten Kapitel zunächst die Gründe und Ziele einer sozialwissenschaftlich gebildeten Persönlichkeit dargelegt (1.1), gegenwärtig besonders bedeutsame Themen für die sozialwissenschaftliche Bildung identifiziert (1.2) und ein integratives Konzept sozialwissenschaftlicher Bildung präsentiert (1.3). Kapitel 2 widmet sich sodann den Herausforderungen, Charakteristika und Kontexten sozialwissenschaftlicher Bildung. Abgesehen von der Diskussion um fachdidaktische Bezugsdisziplinen und der Klärung des Verhältnisses von Fachwissenschaften und -didaktik (2.1) finden sich hier auch Ausführungen zur Stellung und Bedeutung schulischer sozialwissenschaftlicher Bildung (2.2) sowie - durchaus innovativ - die Thematisierung sozialer Ungleichheit und diskriminierender Strukturen in der sozialwissenschaftlichen Bildung selbst (2.3). Die Leitmotive einer solchen Bildung werden schließlich im dritten und zentralen Kapitel des Bandes besprochen. Bemerkenswert ist dessen Architektur, die gewissermaßen einer pädagogischen Doppeldeckerstruktur folgt. So werden zentrale fachdidaktische Prinzipien hier nicht etwa - wie das Inhaltsverzeichnis vermuten lässt - nacheinander durchdekliniert. Stattdessen erfolgt deren Darlegung ,,problemstatt disziplinorientiert" (S. 16) und damit ganz so, wie auch sozialwissenschaftlich Lehrende unterrichten sollten. Gewissermaßen folgerichtig werden in Kapitel 4 sodann zentrale Erkenntnis-, Lehr- und Lernmethoden des sozialwissenschaftlichen Unterrichts erörtert, deren Auswahl sich daran orientiert, dass mittels ihres Einsatzes ein handlungs-, interaktions-, kooperations- und urteilsorientierter sozialwissenschaftlicher Unterricht arrangiert werden kann (S. 133). Dass im Zuge dieser Besprechung jeweils auch Bildungs- und Lernziele sowie mögliche Unterrichtsthemen berücksichtigt werden, ist besonders positiv hervorzuheben. Kapitel 5 widmet sich schließlich der Planung des sozialwissenschaftlichen Unterrichts und liefert dafür hilfreiche theoretische Überlegungen sowie praktische Anregungen. Wie bei allen anderen Kapiteln finden sich auch am Ende dieses Kapitels zahlreiche Denkanstöße, die - allein oder in Lerngruppen - eine Wiederholung zentraler Aspekte, deren Vertiefung und/oder die Reflexion des fachdidaktischen Handelns der Leser*innen ermöglichen (sollen). Ergänzt wird jedes Kapitel durch einen umfänglichen online verfügbaren Foliensatz, der sich mehr oder minder bruchlos in die universitäre Lehre implementieren lässt. Sach- und Personenregister sowie ein - im Übrigen hervorragendes - Literaturverzeichnis schließen den Band ab. Die große Leistung seiner Autor*innen liegt zweifelsfrei darin, dass es ihnen gelingt, den zahlreichen, durchaus ambitionierten Ansprüchen weitestgehend zu genügen. So stiftet ihr durchweg leser*innenfreundlich formuliertes Buch Orientierung, lädt zum Nach-, Über- und Weiterdenken ein, liefert eine große Anzahl unterrichtspraktischer Überlegungen, Hinweise, Materialien usw. - und geht dabei dennoch nicht an den Bedürfnissen der Hochschullehrenden vorbei. Freilich: Angesichts der immensen Aufgaben, die sich Engartner, Hedtke und Zurstrassen gestellt haben, offenbart sich auch die eine oder andere (kleinere) Schwachstelle. So ist $\mathrm{m}$. E. etwa nicht ersichtlich, was es mit dem Neologismus „Fachdidaktikwissenschaftler*in“ auf sich haben soll (Fachdidaktik ist Wissenschaft!) und auch die Auswahl der in Kapitel 5 besprochenen Unterrichtsgegenstände erscheint mir etwas zu beliebig, zumal die Autor*innen zu Beginn 
ihres Bandes selbst zentrale Herausforderungen nennen, von denen sich „,besonders bedeutsame Themen für die sozialwissenschaftliche Bildung“ (S. 18) ablesen lassen. Der wohl streitbarste Punkt ihrer Publikation dürfte allerdings der sein, welche Disziplinen sie in den Reigen der Sozialwissenschaften aufgenommen haben und welche eben nicht. Sicher: Neben Politikwissenschaft und Soziologie, die empirisch in jedem Fall den Kern bilden, auch die Ökonomie aufzunehmen, ist - zumal im Lichte disziplinspezifischer Agenden und bildungspolitisch fragwürdiger Einlassungen - durchaus nachvollziehbar. Gleichermaßen plausibel ist es, nicht noch beliebig viele weitere Disziplinen, wie bspw. die Rechts- und Geschichtswissenschaft oder die Geografie, in einer Art Megasozialwissenschaft zu fusionieren. Die Begründung für ihren Zuschnitt hätte seitens Engartner, Hedtke und Zurstrassen aber durchaus etwas ausführlicher ausfallen dürfen als dies hier (insbesondere S. 13-16) der Fall ist. Positiv gewendet verweist dieser Kritikpunkt aber gleichwohl auf ein hier abermals zu explizierendes Potenzial des Bandes: die ernst zu nehmende Einladung seiner Autor*innen zu einem Nachdenken über eine sozialwissenschaftliche Didaktik, die ihren Namen tatsächlich verdient. Der Wert ihres Buches für diejenigen, die sich nicht den Luxus metatheoretischer Reflexionen leisten können, sondern die tagtäglich sozialwissenschaftlichen Unterricht planen, durchführen und reflektieren müssen, steht jenseits dieser Einladung völlig außer Frage.

Funding Open Access funding enabled and organized by Projekt DEAL.

Open Access Dieser Artikel wird unter der Creative Commons Namensnennung 4.0 International Lizenz veröffentlicht, welche die Nutzung, Vervielfältigung, Bearbeitung, Verbreitung und Wiedergabe in jeglichem Medium und Format erlaubt, sofern Sie den/die ursprünglichen Autor(en) und die Quelle ordnungsgemäß nennen, einen Link zur Creative Commons Lizenz beifügen und angeben, ob Änderungen vorgenommen wurden.

Die in diesem Artikel enthaltenen Bilder und sonstiges Drittmaterial unterliegen ebenfalls der genannten Creative Commons Lizenz, sofern sich aus der Abbildungslegende nichts anderes ergibt. Sofern das betreffende Material nicht unter der genannten Creative Commons Lizenz steht und die betreffende Handlung nicht nach gesetzlichen Vorschriften erlaubt ist, ist für die oben aufgeführten Weiterverwendungen des Materials die Einwilligung des jeweiligen Rechteinhabers einzuholen.

Weitere Details zur Lizenz entnehmen Sie bitte der Lizenzinformation auf http://creativecommons.org/ licenses/by/4.0/deed.de. 